\title{
Synthesis and Characterization of Single Crystalline Silicon Nanowires
}

\author{
Jun Jiao, Devon McClain, Lifeng Dong, and Raj Solanki \\ Department of Physics, Portland State University, Portland, OR 97207
}

Silicon nanowires (SiNWs) have attracted much attention in recent years because they exhibit different properties in comparison to their bulk counterparts. Although they are considered one dimensional nanostrucutres, SiNWs show a variety of morphologies that largely depend on synthesis methods and growth conditions [1]. Several studies have demonstrated that the electronic and optical properties of SiNWs are very much morphology dependent [2-3]. We report here the effect of growing time on the formation of SiNWs in relation to their morphologies and compositions. The characterization efforts include field emission electron microscopy (FESEM), high resolution transmission electron microscopy (HRTEM) and energy dispersive $\mathrm{x}$-ray spectroscopy (EDS).

The SiNWs used in this study were prepared by a chemical vapor deposition (CVD) method Glass substrates with a $250 \mathrm{~nm}$ thick layer of electrically conductive ITO were loaded inside of a quartz horizontal furnace tube and no other catalysts were used. The precursor (10\% disilane, balance Ar) was flowed through the reactor at a rate of $150 \mathrm{sccm}$. The reactor pressure was 400 milliTorrs and the temperature used for growth was $450{ }^{\circ} \mathrm{C}$. When the precursor was not in use, a constant flow of dry $\mathrm{N}_{2}$ through the reactor was maintained. SiNWs samples with different growing times were synthesized. Fig. 1-3 shows a set of FESEM images of the SiNWs samples with growing times of 5, 10 and 20 minutes, respectively. Among the three samples, note that the nanowires formed with the 20 minute reaction time show much larger diameter and longer length, while the SiNWs synthesized with 5 minute reaction time display relatively shorter length and thinner diameter. All three samples show a high yield of SiNWs with rather uniform diameters. Fig. 4 is a HRTEM image of a SiNW taken from the sample with 5 minute growing time. The inset is the diffraction pattern of the same nanowire. Both the lattice fringes of the HRTEM image and the diffraction pattern suggest that the SiNW is single crystalline. A systematic HRTEM characterization of the three samples confirms that the SiNWs are all single crystalline and most are covered by a thin amorphous oxide layer. The chemical composition of the nanowires was analyzed by EDS with the microscope operated in the scanning transmission electron microscopy (STEM) mode. This allows us to use a much finer probe for the single nanowire analysis. Fig. 5 (a) demonstrates a STEM image of a single SiNW with a diameter of about $15 \mathrm{~nm}$. The white circle indicates the location from which the EDS spectrum [as shown in Fig. 5 (b)] was taken. Note in Fig. 5 (b) that the strong Si signals suggest that the SiNW is single crystalline silicon rather than silicon dioxide. The weak O signals in the spectrum confirm the HRTEM observation that there is a thin oxide layer covering the SiNW surface. The signals of $\mathrm{C}$ and $\mathrm{Cu}$ in the spectrum are generated from the holey carbon copper grid used to hold the SiNWs for TEM characterization. Further study is underway to investigate the correlations between the SiNW morphologies and each preparation parameter and their corresponding electronic and optical properties.

\section{References}

[1] Y. H. Tang, Y. F. Zhang, N. Wang, C. S. Lee, X. D. Han, I. Bello, and S. T. Lee, J. Appl. Phys. 85, 7981 (1999).

[2] H. Y. Peng, N. Wang, W. S. Shi, Y. F. Zhang, C. S. Lee, and S. T. Lee, J. Appl. Phys. 89, 727 (2001).

[3] H. Kohno and S. Takeda, Appl. Phys. Lett. 73, 3144 (1998). 

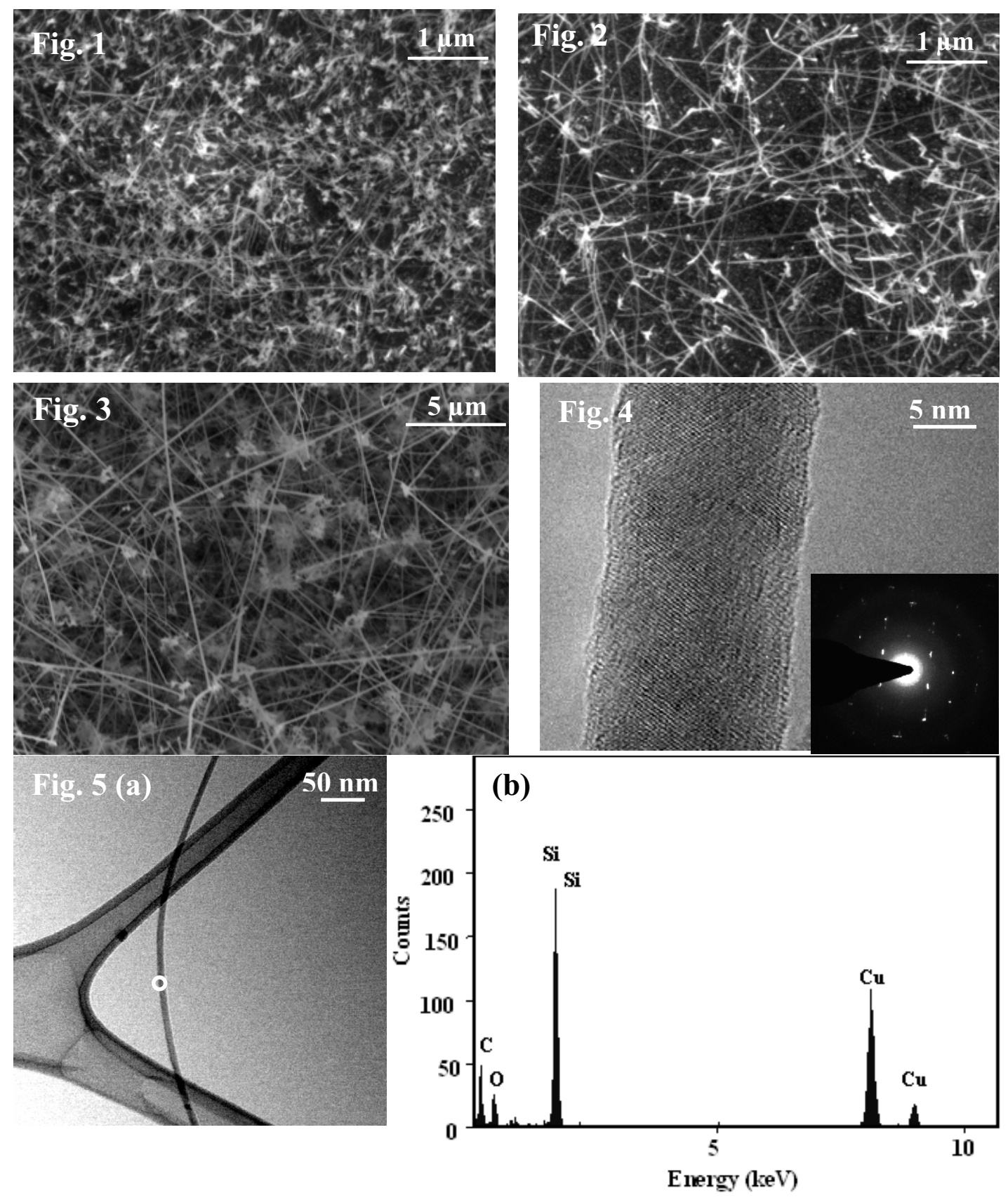

Fig. 1-3 shows a set of FESEM images for the SiNWs samples prepared with growing times of 5, 10 and 20 minutes, respectively.

Fig. 4 is a HRTEM image of SiNW taken from the sample with 5 minutes growing time. The insert is the diffraction pattern of the sample nanowire.

Fig. 5 (a) demonstrates a STEM image of a SiNW with a diameter about $15 \mathrm{~nm}$. Fig. 5 (b) displays the EDS spectrum taken from the location indicated by a white circle in Fig. 5 (a).

Acknowledgement

Financial support for this study was provided in part by the NSF under awards No. ECS-0217061 and No. DMR-0353738. 